\title{
SOME COMMON FIXED POINT THEOREMS FOR ĆIRIĆ TYPE CONTRACTION MAPPINGS
}

\author{
SUMIT CHANDOK
}

\begin{abstract}
Some common fixed point theorems for Ćirić type contraction mappings have been obtained in convex metric spaces. As applications, invariant approximation results for these type of mappings are obtained. The proved results generalize, unify and extend some of the results of the literature.
\end{abstract}

\section{Introduction and preliminaries}

In 1986, Fisher and Sessa [8] obtained the following generalization of a theorem of Gregus [10].

Theorem 1.1. Let $T, I: K \rightarrow K$ be two weakly commuting mappings on a closed convex subset $K$ of a Banach space $X$ satisfying

$$
\|T x-T y\| \leq a\|I x-I y\|+(1-a) \max \{\|I x-T x\|,\|I y-T y\|\}
$$

for all $x, y \in K$, where $0<a<1$. If I is linear, nonexpansive in $K$ such that $T(K) \subseteq I(K)$, then $T$ and I have a unique common fixed point in $K$.

If $I$ is an identity map, we have an immediate generalization of the Gregus fixed point theorem. Mukherjee and Verma [17] generalized Theorem 1.1 by replacing the linearity of $I$ with a more general condition that $I$ is affine, while Jungck [14] generalised it further by replacing commutativity and nonexpansiveness assumptions with compatibility and continuity respectively. Later, many results which are closely related to Gregus's Theorem have appeared in literature (see e.g. [3], [4], [5], [6], [7], [8], [13], [14], [17]). The purpose of this paper is to prove similar type of results for Ćirić type contraction mappings when the underlying spaces are convex metric spaces. Our technique, which is originally due to Gregus [10], has been used by many authors. As applications, common fixed points and invariant approximation results for compatible and $C_{q}$-commuting mappings are obtained. Our results extend

2000 Mathematics Subject Classification. 41A50, 47H10, 54H25.

Key words and phrases. $C_{q}$-commuting, convex metric space, starshaped set, commuting, compatible maps. 
and generalize some of the results of Al-Thagafi [1], Al-Thagafi and Shahzad [2], Babu and Prasad [3], Chandok and Narang [4], Ćirić [5], [6], Diviccaro, Fisher and Sessa [7], Fisher and Sessa [8], Gregus [10], Habiniak [12], Hussain, Rhoades and Jungck [13], Jungck [14], Jungck and Sessa [16], Mukherjee and Verma [17], Narang and Chandok [18], [19], [20], Sahab, Khan and Sessa [21], Shahzad [22], [23], Singh [24], Smoluk [25], Subrahmanyam [26] and of few others.

To begin with, we recall some definitions and known facts to be used in the sequel.

For a metric space $(X, d)$, a continuous mapping $W: X \times X \times[0,1] \rightarrow X$ is said to be (s.t.b.) a convex structure on $X$ if for all $x, y \in X$ and $\lambda \in[0,1]$,

$$
d(u, W(x, y, \lambda)) \leq \lambda d(u, x)+(1-\lambda) d(u, y)
$$

holds for all $u \in X$. The metric space $(X, d)$ together with a convex structure is called a convex metric space [27].

A subset $M$ of a convex metric space $(X, d)$ is said to be a convex set [27] if $W(x, y, \lambda) \in M$ for all $x, y \in M$ and $\lambda \in[0,1]$. A set $M$ is said to be $p$-starshaped [9] where $p \in M$, provided $W(x, p, \lambda) \in M$ for all $x \in M$ and $\lambda \in[0,1]$ i.e. if the segment $[p, x]=\{W(x, p, \lambda): 0 \leq \lambda \leq 1\}$ joining $p$ to $x$ is contained in $M$ for all $x \in M$. $M$ is said to be starshaped if it is $p$-starshaped for some $p \in M$.

Clearly, each convex set $M$ is starshaped but converse is not true.

A convex metric space $(X, d)$ is said to satisfy Property (I) [9] if for all $x, y, q \in X$ and $\lambda \in$ $[0,1]$

$$
d(W(x, q, \lambda), W(y, q, \lambda)) \leq \lambda d(x, y) .
$$

A normed linear space and each of its convex subsets are simple examples of convex metric spaces with $W$ given by $W(x, y, \lambda)=\lambda x+(1-\lambda) y$ for $x, y \in X$ and $0 \leq \lambda \leq 1$. There are many convex metric spaces which are not normed linear spaces (see [9], [27]). Property (I) is always satisfied in a normed linear space.

For a non-empty subset $M$ of a metric space $(X, d)$ and $x \in X$, an element $y \in M$ is s.t.b. a best approximant to $x$ or a best $M$-approximant to $x$ if $d(x, y)=d(x, M) \equiv \inf \{d(x, y): y \in M\}$. The set of all such $y \in M$ is denoted by $P_{M}(x)$.

For a convex subset $M$ of a convex metric space $(X, d)$, a mapping $g: M \rightarrow X$ is s.t.b. affine if for all $x, y \in M, g(W(x, y, \lambda))=W(g x, g y, \lambda)$ for all $\lambda \in[0,1] . g$ is s.t.b. affine with respect to $p \in M$ if $g(W(x, p, \lambda))=W(g x, g p, \lambda)$ for all $x \in M$ and $\lambda \in[0,1]$.

Suppose $(X, d)$ is a metric space, $M$ a nonempty subset of $X$, and $S, T$ be self mappings of $M . T$ is s.t.b.

(i) $S$-contraction if there exists a $k \in[0,1)$ such that $d(T x, T y) \leq k d(S x, S y)$, 
(ii) $S$-nonexpansive if $d(T x, T y) \leq d(S x, S y)$ for all $x, y \in M$.

If $S=$ identity mapping, then $T$ is s.t.b. a contraction (nonexpansive, respectively).

Let $M$ a nonempty subset of a metric space $(X, d)$, a point $x \in M$ is a common fixed (coincidence) point of $S$ and $T$ if $x=S x=T x(S x=T x)$. The set of fixed points (respectively, coincidence points) of $S$ and $T$ is denoted by $F(S, T)$ (respectively, $C(S, T)$ ). Then mappings $T, S: M \rightarrow M$ are s.t.b.

(i) commuting on $M$ if $S T x=T S x$ for all $x \in M$;

(ii) $R$-weakly commuting on $M$ if there exists $R>0$ such that $d(T S x, S T x) \leq R d(T x, S x)$ for all $x \in M$.

If $R=1$, then the maps are called weakly commuting.

(iii) compatible if $\lim d\left(T S x_{n}, S T x_{n}\right)=0$ whenever $\left\{x_{n}\right\}$ is a sequence such that $\lim T x_{n}=$ $\lim S x_{n}=t$ for some $t$ in $M$.

(iv) weakly compatible if they commute at their coincidence points,i.e., if $S T x=T S x$ whenever $S x=T x$.

Suppose $(X, d)$ is a convex metric space, $M$ a $q$-starshaped subset with $q \in F(S) \cap M$ and is both $T$ - and $S$-invariant. Then $T$ and $S$ are called

(i) $R$-subweakly commuting on $M$ if for all $x \in M$, there exists a real number $R>0$ such that $d(T S x, S T x) \leq R \operatorname{dist}(S x, W(T x, q, k)), k \in[0,1]$

(ii) $C_{q}$-commuting if $S T x=T S x$ for all $x \in C_{q}(S, T)$, where $C_{q}(S, T)=\cup\left\{C\left(S, T_{k}\right): 0 \leq k \leq 1\right\}$ and $T_{k} x=\{W(T x, q, k): 0 \leq k \leq 1\}$.

$C_{q}$-commuting maps are weakly compatible. However, converse is not true.

Example 1.2 ([2]). Let $X=\mathbb{R}$ be endowed with the usual metric and $M=[0, \infty)$. Define $T, S$ : $M \rightarrow M$ by $T x=x^{2}$ for all $x \neq 2$ and $T 2=1$; and $S x=2 x$ for all $x \in M$. Then $M$ is $q$-starshaped with $q=0, C(T, S)=\{0\}$ and $C_{q}(T, S)=\{0\} \cup[2, \infty)$. Moreover, $T$ and $S$ are weakly compatible but not $C_{q}$-commuting.

Commuting mappings are R-subweakly commuting, but the converse may not be true (see [23]). It is well known that R-subweakly commuting maps are R-weakly commuting but not conversely (see [22]). R-subweakly commuting maps are weakly compatible but the converse does not hold (see [22], [23]).

R-subweakly commuting maps are $C_{q}$-commuting but converse does not hold.

Example 1.3 ([2]). Let $X=\mathbb{R}$ be endowed with the usual metric and $M=[0, \infty)$. Define $T, S$ : $M \rightarrow M$ by $T x=\frac{1}{2}$ if $0 \leq x<1$ and $T x=x^{2}$ if $x \geq 1$; and $S x=\frac{x}{2}$ if $0 \leq x<1$ and $S x=x$ if 
$x \geq 1$. Then $M$ is $q$-starshaped with $q=1$, and $C_{q}(T, S)=[1, \infty)$. Moreover $S$ and $T$ are $C_{q^{-}}$ commuting but neither $R$-weakly commuting nor $R$-subweakly commuting for all $R>0$.

\section{Main results}

We begin the section with the following result which extends and generalizes the corresponding results of [3], [4], [5], [6], [7], [8], [13], [14] and [17].

Theorem 2.1. Let $M$ be a nonempty closed convex subset of a complete convex metric space $(X, d)$. Let $f, T: M \rightarrow M$ self mappings, and $c l T(M) \subseteq f(M)$. Suppose that $f, T$ satisfies

$$
d(T x, T y) \leq a \max \{d(f x, f y), c\{d(f x, T y)+d(f y, T x)\}\}+b \max \{d(f x, T x), d(f y, T y)\}
$$

for all $x, y \in M$, where $0<a<1, b \geq 0, a+b=1$ and $0 \leq c<\eta, \eta=\min \left\{\frac{2+a}{5+a}, \frac{2-a}{4}, \frac{4}{9+a}\right\}<\frac{1}{2}$. Further, if $f$ and $T$ are weakly compatible on $M$ and $f$ is affine, then $F(f) \cap F(T)$ is singleton.

Proof. Let $x=x_{\circ}$ be an arbitrary point of $M$. Let $x_{1}, x_{2}, x_{3}$ be points in $M$ such that $f x_{1}=T x$, $f x_{2}=T x_{1}, f x_{3}=T x_{2}$, so that $T x_{r-1}=f x_{r}$, for $r=1,2,3$, as $T(M) \subseteq c l T(M) \subseteq f(M)$.

$$
\begin{aligned}
d\left(T x_{r}, f x_{r}\right)= & d\left(T x_{r}, T x_{r-1}\right) \\
\leq & a \max \left\{d\left(f x_{r}, f x_{r-1}\right), c\left\{d\left(f x_{r}, T x_{r-1}\right)+d\left(f x_{r-1}, T x_{r}\right)\right\}\right\} \\
& +b \max \left\{d\left(f x_{r}, T x_{r}\right), d\left(f x_{r-1}, T x_{r-1}\right)\right\} \\
\leq & a \max \left\{d\left(T x_{r-1}, f x_{r-1}\right), c\left\{d\left(f x_{r}, f x_{r}\right)+d\left(f x_{r-1}, T x_{r-1}\right)+d\left(T x_{r-1}, T x_{r}\right)\right\}\right\} \\
& +b \max \left\{d\left(f x_{r}, T x_{r}\right), d\left(f x_{r-1}, T x_{r-1}\right)\right\} .
\end{aligned}
$$

If $d\left(T x_{r-1}, f x_{r-1}\right)<d\left(T x_{r}, f x_{r}\right)$, then we have

$$
\begin{aligned}
d\left(T x_{r}, f x_{r}\right) & <a \max \left\{d\left(T x_{r}, f x_{r}\right), 2 c d\left(f x_{r}, T x_{r}\right)\right\}+b d\left(f x_{r}, T x_{r}\right) \\
& =(a+b) d\left(T x_{r}, f x_{r}\right),
\end{aligned}
$$

a contradiction. Thus, we have

$$
d\left(T x_{r}, f x_{r}\right) \leq d\left(T x_{r-1}, f x_{r-1}\right) \leq d\left(T x_{0}, f x_{0}\right) .
$$

So, it follows that

$$
\begin{aligned}
d\left(T x_{2}, f x_{1}\right)= & d\left(T x_{2}, T x_{0}\right) \\
\leq & a \max \left\{d\left(f x_{2}, f x_{0}\right), c\left\{d\left(f x_{2}, T x_{0}\right)+d\left(f x_{0}, T x_{2}\right)\right\}\right\} \\
& +b \max \left\{d\left(f x_{2}, T x_{2}\right), d\left(f x_{0}, T x_{0}\right)\right\} \\
\leq & a \max \left\{d\left(f x_{2}, f x_{1}\right)+d\left(f x_{1}, f x_{0}\right), c\left\{d\left(f x_{2}, T x_{0}\right)+d\left(f x_{0}, f x_{1}\right)\right.\right.
\end{aligned}
$$




$$
\begin{aligned}
& \left.\left.+d\left(f x_{1}, T x_{1}\right)+d\left(T x_{1}, T x_{2}\right)\right\}\right\}+b \max \left\{d\left(f x_{2}, T x_{2}\right), d\left(f x_{0}, T x_{0}\right)\right\} \\
= & a \max \left\{d\left(T x_{1}, f x_{1}\right)+d\left(T x_{0}, f x_{0}\right), c\left\{d\left(T x_{1}, f x_{1}\right)+d\left(f x_{0}, T x_{0}\right)\right.\right. \\
& \left.\left.+d\left(f x_{1}, T x_{1}\right)+d\left(f x_{2}, T x_{2}\right)\right\}\right\}+b \max \left\{d\left(f x_{2}, T x_{2}\right), d\left(f x_{0}, T x_{0}\right)\right\} \\
\leq & a \max \left\{d\left(T x_{0}, f x_{0}\right)+d\left(T x_{0}, f x_{0}\right), c\left\{d\left(T x_{0}, f x_{0}\right)+d\left(f x_{0}, T x_{0}\right)\right.\right. \\
& \left.\left.+d\left(f x_{0}, T x_{0}\right)+d\left(f x_{0}, T x_{0}\right)\right\}\right\}+b \max \left\{d\left(f x_{0}, T x_{0}\right), d\left(f x_{0}, T x_{0}\right)\right\} \\
= & a \max \left\{2 d\left(T x_{0}, f x_{0}\right), 4 c d\left(T x_{0}, f x_{0}\right)\right\}+b d\left(f x_{0}, T x_{0}\right) \\
= & (2 a+b) d\left(T x_{0}, f x_{0}\right) \\
= & (1+a) d\left(T x_{0}, f x_{0}\right) .
\end{aligned}
$$

Hence

$$
d\left(T x_{2}, f x_{1}\right)=d\left(T x_{2}, T x_{0}\right) \leq(1+a) d\left(T x_{0}, f x_{0}\right) .
$$

Let $z=W\left(x_{2}, x_{3}, \frac{1}{2}\right)$. Since $C$ is convex and $f$ is affine, $f z=f W\left(x_{2}, x_{3}, \frac{1}{2}\right)=W\left(f x_{2}, f x_{3}, \frac{1}{2}\right)=$ $W\left(T x_{1}, T x_{2}, \frac{1}{2}\right)$. Therefore,

$$
\begin{aligned}
d\left(f z, f x_{1}\right) & =d\left(W\left(T x_{1}, T x_{2}, \frac{1}{2}\right), T x_{0}\right) \\
\leq & \frac{1}{2} d\left(T x_{1}, T x_{0}\right)+\frac{1}{2} d\left(T x_{2}, T x_{0}\right) \\
\leq & \frac{1}{2}\left[d\left(T x_{1}, f x_{1}\right)+(1+a) d\left(T x_{0}, f x_{0}\right)\right] \\
\leq & \frac{1}{2}\left[d\left(T x_{0}, f x_{0}\right)+(1+a) d\left(T x_{0}, f x_{0}\right)\right] \\
= & \left(1+\frac{a}{2}\right) d\left(T x_{0}, f x_{0}\right), \\
d\left(f z, f x_{2}\right) & =d\left(W\left(T x_{1}, T x_{2}, \frac{1}{2}\right), T x_{1}\right) \\
& \leq \frac{1}{2} d\left(T x_{1}, T x_{1}\right)+\frac{1}{2} d\left(T x_{2}, T x_{1}\right) \\
& \leq \frac{1}{2} d\left(T x_{0}, f x_{0}\right) . \\
d\left(f z, f x_{3}\right) & =d\left(W\left(T x_{1}, T x_{2}, \frac{1}{2}\right), T x_{2}\right) \\
& \leq \frac{1}{2} d\left(T x_{1}, T x_{2}\right)+\frac{1}{2} d\left(T x_{2}, T x_{2}\right) \\
& \leq \frac{1}{2} d\left(T x_{0}, f x_{0}\right) .
\end{aligned}
$$

Assume that $M=\max \left\{d(f z, T z), d\left(T x_{0}, f x_{0}\right)\right\}$. Consider $d(T z, f z)=d\left(T z, W\left(T x_{1}, T x_{2}, \frac{1}{2}\right)\right)$ 


$$
\begin{aligned}
\leq & \frac{1}{2} d\left(T z, T x_{1}\right)+\frac{1}{2} d\left(T z, T x_{2}\right) \\
\leq & \frac{1}{2}\left[a \max \left\{d\left(f z, f x_{1}\right), c\left\{d\left(f z, T x_{1}\right)+d\left(f x_{1}, T z\right)\right\}\right\}\right. \\
& \left.+b \max \left\{d(f z, T z), d\left(f x_{1}, T x_{1}\right)\right\}\right]+\frac{1}{2}\left[a \max \left\{d\left(f z, f x_{2}\right), c\left\{d\left(f z, T x_{2}\right)+d\left(f x_{2}, T z\right)\right\}\right\}\right. \\
& \left.+b \max \left\{d(f z, T z), d\left(f x_{2}, T x_{2}\right)\right\}\right] \\
\leq & \frac{1}{2}\left[a \max \left\{d\left(f z, f x_{1}\right), c\left\{d\left(f z, T x_{1}\right)+d\left(f x_{1}, f z\right)+d(f z, T z)\right\}\right\}+b M\right] \\
& +\frac{1}{2}\left[a \max \left\{d\left(f z, f x_{2}\right), c\left\{d\left(f z, T x_{2}\right)+d\left(f x_{2}, f z\right)+d(f z, T z)\right\}\right\}+b M\right] \\
\leq & \frac{1}{2}\left[a \max \left\{d\left(f z, f x_{1}\right), c\left\{d\left(f z, f x_{2}\right)+d\left(f x_{1}, f z\right)+d(f z, T z)\right\}\right\}+b M\right] \\
& +\frac{1}{2}\left[a \max \left\{d\left(f z, f x_{2}\right), c\left\{d\left(f z, T x_{2}\right)+d\left(f x_{2}, f z\right)+d(f z, T z)\right\}\right\}+b M\right] \\
\leq & \frac{1}{2}\left[a \max \left\{\left(1+\frac{a}{2}\right) d\left(T x_{0}, f x_{0}\right), c\left\{\frac{1}{2} d\left(T x_{0}, f x_{0}\right)+\left(1+\frac{a}{2}\right) d\left(T x_{0}, f x_{0}\right)+d(f z, T z)\right\}\right\}+b M\right] \\
& +\frac{1}{2}\left[a \max \left\{\frac{1}{2} d\left(T x_{0}, f x_{0}\right), c\left\{\frac{1}{2} d\left(T x_{0}, f x_{0}\right)+\frac{1}{2} d\left(T x_{0}, f x_{0}\right)+d(f z, T z)\right\}\right\}+b M\right] \\
\leq & \frac{1}{2}\left[a \max \left\{\left(1+\frac{a}{2}\right) M, c\left\{\frac{5+a}{2} M\right\}\right\}+b M\right]+\frac{1}{2}\left[a \max \left\{\frac{1}{2} M, 2 c M\right\}+b M\right] \\
= & \frac{1}{2}\left[a \max \left\{\left(1+\frac{a}{2}\right) M, c\left\{\frac{5+a}{2} M\right\}\right\}\right]+\frac{1}{2}\left[a \max \left\{\frac{1}{2} M, 2 c M\right\}\right]+b M
\end{aligned}
$$

Now the following four possible cases may arise.

Case 1. If $\max \left\{\left(1+\frac{a}{2}\right) M, \frac{5+a}{2} c M\right\}=\left(1+\frac{a}{2}\right) M$ and $\max \left\{\frac{1}{2} M, 2 c M\right\}=\frac{1}{2} M$, we have

$$
\begin{aligned}
d(T z, f z) & \leq \frac{1}{2}\left[a\left(1+\frac{a}{2}\right) M\right]+\frac{1}{2}\left[a \frac{1}{2} M\right]+b M \\
& =\left[\frac{1}{4}\{a(a+2)+a\}+(1-a)\right] M \\
& =\lambda_{1} M,
\end{aligned}
$$

where $\lambda_{1}=\frac{a^{2}-a+4}{4}<1$.

Case 2. If $\max \left\{\left(1+\frac{a}{2}\right) M, \frac{5+a}{2} c M\right\}=\left(1+\frac{a}{2}\right) M$ and $\max \left\{\frac{1}{2} M, 2 c M\right\}=2 c M$, we have

$$
\begin{aligned}
d(T z, f z) & \leq \frac{1}{2}\left[a\left(1+\frac{a}{2}\right) M\right]+\frac{1}{2}[a 2 c M]+b M \\
& =\left[\frac{1}{4}\{a(a+2)+4 a c\}+(1-a)\right] M \\
& =\lambda_{2} M,
\end{aligned}
$$

where $\lambda_{2}=\frac{a^{2}-2 a+4 a c+4}{4}<1$.

Case 3. If $\max \left\{\left(1+\frac{a}{2}\right) M, \frac{5+a}{2} c M\right\}=\frac{5+a}{2} c M$ and $\max \left\{\frac{1}{2} M, 2 c M\right\}=2 c M$, we have

$$
d(T z, f z) \leq \frac{1}{2}\left[a \frac{5+a}{2} c M\right]+\frac{1}{2}[a 2 c M]+b M
$$




$$
\begin{aligned}
& =\left[\frac{1}{4}\{a(a+5) c+4 a c\}+(1-a)\right] M \\
& =\lambda_{3} M,
\end{aligned}
$$

where $\lambda_{3}=\frac{a^{2} c-4 a+9 a c+4}{4}<1$.

Case 4. If $\max \left\{\left(1+\frac{a}{2}\right) M, \frac{5+a}{2} c M\right\}=\frac{5+a}{2} c M$ and $\max \left\{\frac{1}{2} M, 2 c M\right\}=\frac{1}{2} M$, it follows that $\frac{2+a}{5+a} \leq c \leq$ $\frac{1}{4}$, and since $c \leq \eta \leq \frac{2+a}{5+a}$. So this case does not arise, and so from the above cases we have

$$
d(T z, f z) \leq \lambda M
$$

where $\lambda=\max \left\{\lambda_{1}, \lambda_{2}, \lambda_{3}\right\}<1$.

Thus it follows that

$$
\begin{aligned}
d(T z, f z) & \leq \lambda \max \left\{d(f z, T z), d\left(T x_{0}, f x_{0}\right)\right\} \\
& \leq \lambda d\left(T x_{0}, f x_{0}\right)
\end{aligned}
$$

We therefore have $\inf \left\{d(T z, f z): z=W\left(x_{2}, x_{3}, \frac{1}{2}\right\} \leq \lambda \inf \{d(T x, f x): x \in C\}\right.$ and since $\inf \left\{d(T z, f z): z=W\left(x_{2}, x_{3}, \frac{1}{2}\right\} \geq \inf \{d(T x, f x): x \in C\}\right.$, it follows that $\inf \{d(T x, f x): x \in C\}=0$. Then the sets defined by $K_{n}=\left\{x \in C: d(T x, f x) \leq \frac{1}{n}\right\}$, for $n=1,2, \ldots$ must be nonempty and $K_{1} \supseteq K_{2} \supseteq \ldots \supseteq K_{n} \supseteq \ldots$. Thus $\operatorname{cl}\left(T K_{n}\right)$ is nonempty for $n=1,2 \ldots$ and $\operatorname{cl}\left(T K_{1}\right) \supseteq \operatorname{cl}\left(T K_{2}\right) \supseteq$ $\ldots \supseteq \operatorname{cl}\left(T K_{n}\right) \supseteq \ldots$... Further, for all $x, y \in K_{n}$,

$$
\begin{aligned}
d(T x, T y) \leq & a \max \{d(f x, f y), c\{d(f x, T y)+d(f y, T x)\}\}+b \max \{d(f x, T x), d(f y, T y)\} \\
\leq & a \max \{d(f x, T x)+d(T x, T y)+d(T y, f y), c\{d(f x, T x)+d(T x, T y) \\
& +d(f y, T y)+d(T y, T x)\}\}+b \max \{d(f x, T x), d(f y, T y)\} \\
\leq & a \max \left\{\frac{1}{n}+d(T x, T y)+\frac{1}{n}, c\left\{\frac{1}{n}+d(T x, T y)+\frac{1}{n}+d(T y, T x)\right\}\right\}+b \max \left\{\frac{1}{n}, \frac{1}{n}\right\} \\
\leq & a \max \left\{\frac{2}{n}+d(T x, T y), c\left\{\frac{2}{n}+2 d(T x, T y)\right\}\right\}+b \frac{1}{n} .
\end{aligned}
$$

Case 1. If $\max \left\{\frac{2}{n}+d(T x, T y), c\left\{\frac{2}{n}+2 d(T x, T y)\right\}\right\}=\frac{2}{n}+d(T x, T y)$, we have

$$
\begin{aligned}
d(T x, T y) & \leq a\left\{\frac{2}{n}+d(T x, T y)\right\}+b \frac{1}{n} \\
& =\frac{2 a+b}{n}+a d(T x, T y),
\end{aligned}
$$

which implies that $d(T x, T y) \leq \frac{a+1}{(1-a) n}$.

Case 2. If $\max \left\{\frac{2}{n}+d(T x, T y), c\left\{\frac{2}{n}+2 d(T x, T y)\right\}\right\}=c\left\{\frac{2}{n}+2 d(T x, T y)\right\}$, we have

$$
d(T x, T y) \leq a c\left\{\frac{2}{n}+2 d(T x, T y)\right\}+b \frac{1}{n}
$$




$$
\begin{aligned}
& =2 a c\left\{\frac{1}{n}+d(T x, T y)\right\}+b \frac{1}{n} \\
& <a\left\{\frac{1}{n}+d(T x, T y)\right\}+b \frac{1}{n} \\
& =\frac{1}{n}+a d(T x, T y),
\end{aligned}
$$

which implies that $d(T x, T y)<\frac{1}{(1-a) n} \leq \frac{a+1}{(1-a) n}$.

Thus $\lim \operatorname{diam}\left(T K_{n}\right)=\lim \operatorname{diam}\left(\operatorname{cl}\left(T K_{n}\right)\right)=0$, i.e. $\operatorname{cl}\left(T K_{n}\right)$ is a decreasing sequence of nonempty closed subsets of $M$ whose sequence $\left\{\operatorname{diam}\left(\operatorname{cl}\left(T K_{n}\right)\right)\right\}$ of the diameters converges to zero and by Cantor's Intersection Theorem, $A=\cap_{n=1}^{\infty}\left\{\operatorname{cl}\left(T K_{n}\right): n \in \mathbb{N}\right\}$ is singleton and hence nonempty. If $v \in A$ for each $n$, then there is a $y_{n} \in T K_{n}$ such that $d\left(v, y_{n}\right)<\frac{1}{n}$. Hence for each $n$, there is an $x_{n} \in K_{n}$ such that $y_{n}=T x_{n}$ and $d\left(\nu, T x_{n}\right)<\frac{1}{n}$ for all $n$ and so $T x_{n} \rightarrow v$. Since $x_{n} \in K_{n}$, we have $d\left(f x_{n}, T x_{n}\right) \leq \frac{1}{n}$. Thus $\lim f x_{n}=\lim T x_{n}=v \in \operatorname{cl} T(M) \subseteq f(M)$ which implies that there exists some $q \in M$ such that $v=f q$. Now,

$$
\begin{aligned}
d(\nu, T q) \leq & d\left(\nu, T x_{n}\right)+d\left(T x_{n}, T q\right) \\
\leq & d\left(\nu, T x_{n}\right)+a \max \left\{d\left(f x_{n}, f q\right), c\left\{d\left(f q, T x_{n}\right)+d\left(f x_{n}, T q\right)\right\}\right\} \\
& +b \max \left\{d(f q, T q), d\left(f x_{n}, T x_{n}\right)\right\} \\
\leq & d\left(\nu, T x_{n}\right)+a \max \left\{d\left(f x_{n}, v\right), c\left\{d\left(\nu, T x_{n}\right)+d\left(f x_{n}, T q\right)\right\}\right\} \\
& +b \max \left\{d(\nu, T q), d\left(f x_{n}, T x_{n}\right)\right\} .
\end{aligned}
$$

Taking the limit as $n \rightarrow \infty$, we get

$$
\begin{aligned}
d(\nu, T q) & \leq a c d(\nu, T q)+b d(\nu, T q) \\
& =(a c+b) d(\nu, T q) \\
& =[1-a(1-c)] d(f w, T w) .
\end{aligned}
$$

This implies that $T q=v=f q$. Since $f$ and $T$ are weakly compatible on $M, f T q=T f q$. Thus $f v=T \nu$. Now,

$$
\begin{aligned}
d(v, T v)= & d(T q, T v) \\
\leq & a \max \{d(f q, f v), c\{d(f q, T v)+d(f v, T q)\}\} \\
& +b \max \{d(f q, T q), d(f v, T v)\} \\
= & a \max \{d(v, T v), 2 c d(v, T v)\} \\
\leq & a d(\nu, T v) \\
< & d(v, T v)
\end{aligned}
$$

This implies that $T v=v=f v$. 
Now we prove the uniqueness. Suppose that $v$ and $w$ are common fixed points of $T$ and $f$ i.e., there exists $w \in M$ such that $T w=w=f w$. Then

$$
\begin{aligned}
d(w, v)= & d(T w, T v) \\
\leq & a \max \{d(f w, f v), c\{d(f w, T v)+d(f v, T w)\}\} \\
& +b \max \{d(f w, T w), d(f v, T v)\} \\
= & a \max \{d(\nu, w), 2 c d(\nu, w)\} \\
\leq & a d(v, T v) \\
< & d(\nu, T v) .
\end{aligned}
$$

This gives that $v=w$.

The first part of the above proof gives the following result.

Corollary 2.2. Let $T$ and $f$ be self maps of a closed convex subset $M$ of a complete convex metric space $(X, d)$. Suppose $f$ is affine and $c l T(M) \subseteq f(M)$. If $T$ and $f$ satisfy $(2.1)$, then $T$ and $f$ have a coincidence point in $M$.

Example 2.3. Let $X=\mathbb{R}$ with the usual metric $d(x, y)=|x-y|$. Define self maps $T, f: X \rightarrow X$ by $T x=\frac{2+x}{3}$ and $f x=\frac{3 x-1}{2}, x \in X$. Clearly, $f$ is affine and $T$ and $f$ are weakly compatible mappings on $X$. Now for any $x, y \in X, d(T x, T y)=\left|\frac{x-y}{3}\right|=\frac{2}{9} d(f x, f y)$, so that $T$ and $f$ satisfy the inequality (2.1) with $a=\frac{2}{9}, b=\frac{7}{9}$ and $c \leq \frac{20}{47}$. Thus, all the hypotheses of Theorem 2.1 are satisfied and $\{1\}$ is a unique common fixed point of $T$ and $f$.

Theorem 2.4. Let $M$ be a closed convex subset of a convex metric space $(X, d)$ with Property (I), $f, T$ are self mappings of $M$. Suppose that $c l T(M) \subseteq f(M), f$ is affine w.r.t. $q \in F(f)$. If clT $(M)$ is compact, $T$ is continuous, $(f, T)$ is $C_{q}$-commuting, and satisfies for some $q \in F(f)$,

$$
\begin{aligned}
d(T x, T y) \leq & \max \{d(f x, f y), c[\operatorname{dist}(f x,[q, T y])+\operatorname{dist}(f y,[q, T x])]\}+ \\
& \frac{1-k}{k} \max \{\operatorname{dist}(f x,[q, T x]), \operatorname{dist}(f y,[q, T y])\},
\end{aligned}
$$

for all $x, y \in M, 0 \leq c<\frac{1}{2}, k \in(0,1)$, then $T$ and $f$ have a common fixed point.

Proof. Define $T_{n}: M \rightarrow M$ as $T_{n} x=W\left[T x, q, k_{n}\right]$ for all $x \in M$, for each $n \geq 1$, where $\left\{k_{n}\right\}$ is a sequence of real numbers in $(0,1)$ such that $k_{n} \rightarrow 1$. As $M$ is convex, $q \in F(f)$ and $\operatorname{cl} T(M) \subseteq$ $f(M), T_{n}$ is a self mapping of $M$ and $c l\left[T_{n}(M)\right] \subseteq f(M)$ for each $n$. Since $T$ and $f$ are $C_{q^{-}}$ commuting, $f$ is affine with respect to $q \in F(f)$, it follows for each $x \in C_{q}(f, T), f T_{n} x=$ $f\left(W\left[T x, q, k_{n}\right]\right)=W\left[f T x, f q, k_{n}\right]=W\left[T f x, f q, k_{n}\right]=T_{n} f x$. Thus $f T_{n} x=T_{n} f x$ for each $x \in C\left(f, T_{n}\right) \subseteq C_{q}(f, T)$. Hence the pair $f$ and $T_{n}$ are weakly compatible for all $n$. Further, we have

$$
d\left(T_{n} x, T_{n} y\right)=d\left(W\left[T x, q, k_{n}\right], W\left[T y, q, k_{n}\right]\right)
$$




$$
\begin{aligned}
\leq & k_{n} d(T x, T y) \\
\leq & k_{n}\{\max \{d(f x, f y), c[\operatorname{dist}(f x,[q, T y])+\operatorname{dist}(f y,[q, T x])]\} \\
& \left.+\frac{1-k}{k} \max \{\operatorname{dist}(f x,[q, T x]), \operatorname{dist}(f y,[q, T y])\}\right\} \\
\leq & k_{n} \max \left\{d(f x, f y), c\left[d\left(f x, T_{n} y\right)+d\left(f y, T_{n} x\right)\right]\right\} \\
& +\left(1-k_{n}\right) \max \left\{d\left(f x, T_{n} x\right), d\left(f y, T_{n} y\right)\right\}
\end{aligned}
$$

for all $x, y \in M$ and $0<k_{n}<1$. By Theorem 2.1, for each $n \geq 1$, there exists an $x_{n} \in M$ such that $x_{n}=f x_{n}=T_{n} x_{n}$. The compactness of $c l T(M)$ implies that there exists a subsequence $T x_{n_{i}}$ of $T x_{n}$ such that $T x_{n_{i}} \rightarrow z \in \operatorname{cl} T(M) . x_{n_{i}}=T_{n_{i}} x_{n_{i}}=W\left(T x_{n_{i}}, q, k_{n_{i}}\right) \rightarrow z$. As $T$ is continuous, $T x_{n_{i}} \rightarrow T z$. Thus $z=T z$. As $c l T(M) \subset f(M)$, it follows that $f u=z=T z$ for some $u \in M$ and further

$$
\begin{aligned}
d\left(T x_{n_{i}}, T u\right) \leq & \max \left\{d\left(f x_{n_{i}}, f u\right), c\left[\operatorname{dist}\left(f x_{n_{i}},[q, T u]\right)+\operatorname{dist}\left(f u,\left[q, T x_{n_{i}}\right]\right)\right]\right\} \\
& \left.+\frac{1-k}{k} \max \left\{\operatorname{dist}\left(f x_{n_{i}},\left[q, T x_{n_{i}}\right]\right), \operatorname{dist}(f u,[q, T u])\right\}\right\} \\
\leq & \left.\max \left\{d\left(f x_{n_{i}}, z\right), c\left[d\left(f x_{n_{i}}, T_{n_{i}} u\right]\right)+d\left(z, T_{n_{i}} x_{n_{i}}\right)\right]\right\} \\
& \left.+\frac{1-k_{n_{i}}}{k_{n_{i}}} \max \left\{d\left(f x_{n_{i}}, T_{n_{i}} x_{n_{i}}\right), d\left(z, T_{n_{i}} u\right)\right\}\right\} .
\end{aligned}
$$

On letting $n \rightarrow \infty$, we have $d(z, T u) \rightarrow 0, T u=z=T z=f u$. As $f$ and $T$ are also weakly compatible, we have $f z=f T u=T f u=T z=z$. Hence the result.

Remark 2.1. Theorem 2.4 extends and generalizes Theorem 2.2 of [1] and [2], Theorem 2.3 of [13], Lemma 2.2 of [22] and Theorem 2.1 of [23] to maps satisfying a more general inequality and without linearity, and also when the underlying spaces are convex metric spaces.

The following result will be used in the sequel.

Proposition 2.5. If $M$ is a subset of a convex metric space $(X, d), u \in X$ and $y \in P_{M}(u)$, then the line segment $\{W(y, u, \lambda): 0<\lambda<1\}$ and the set $M$ are disjoint.

Proof. Since $y \in P_{M}(u)$, consider

$$
\begin{aligned}
d(u, W(y, u, \lambda)) & \leq \lambda d(u, y) \\
& <d(u, M) \text {, for every } 0<\lambda<1 .
\end{aligned}
$$

This implies that $W(y, u, \lambda) \notin M$ for any $\lambda, 0<\lambda<1$. Therefore the line segment $\{W(y, u, \lambda)$ : $0<\lambda<1\}$ and the set $M$ are disjoint.

Theorem 2.6. Let $M$ be a subset of a convex metric space $(X, d)$ with Property (I) and $T, S$ are self mappings of $M$ such that $u \in F(S) \cap F(T)$ for some $u \in M$ and $T(\partial M \cap M) \subseteq M$. Suppose that $P_{M}(u)$ is nonempty, closed and convex, $S$ is affine with respect to $q \in F(S), T$ is continuous 
on $P_{M}(u)$ and $\operatorname{cl} T\left(P_{M}(u)\right) \subseteq S\left(P_{M}(u)\right)=P_{M}(u)$. If cl $\left.T\left(P_{M}(u)\right)\right)$ is compact and $(T, S)$ is $C_{q^{-}}$ commuting and satisfies

$$
d(T x, T y) \leq \begin{cases}d(S x, S y), & \text { if } y=u \\ Q(x, y), & \text { if } y \in P_{M}(u),\end{cases}
$$

where

$$
\begin{aligned}
Q(x, y)= & \max \{d(S x, S y), c[\operatorname{dist}(S x,[q, T y])+\operatorname{dist}(S y,[q, T x])]\}+ \\
& \frac{1-k}{k} \max \{\operatorname{dist}(S x,[q, T x]), \operatorname{dist}(S y,[q, T y])\},
\end{aligned}
$$

for $0 \leq c<\frac{1}{2}, k \in(0,1)$, then $P_{M}(u) \cap F(S) \cap F(T) \neq \varnothing$.

Proof. Let $x \in P_{M}(u)$. For any $\lambda \in(0,1)$, we have

$$
d(W(u, x, \lambda), u) \leq \lambda d(u, u)+(1-\lambda) d(x, u)=(1-\lambda) d(x, u)<\operatorname{dist}(u, M) .
$$

It follows from Proposition 2.5 that the open line segment $\{W(u, x, \lambda): 0<\lambda<1\}$ and the set $M$ are disjoint. Thus $x$ is not in the interior of $M$ and so $x \in \partial M \cap M$. Since $T(\partial M \cap M) \subset M$, $T x$ must be in $M$. Also $S x \in P_{M}(u), u \in F(T) \cap F(S)$, and $(T, S)$ satisfy (2.2), we have

$$
d(T x, u)=d(T x, T u) \leq d(S x, S u)=d(S x, u) \leq \operatorname{dist}(u, C)
$$

This implies that $T x \in P_{M}(u)$. Moreover, $\operatorname{cl} T\left(P_{M}(u)\right) \subseteq S\left(P_{M}(u)\right)=P_{M}(u)$. Hence the result follows from Theorem 2.4.

Remark 2.2. Theorem 2.6 extends and generalizes the corresponding results of [2], [4], [13], [16], [20], [21], [24], [25] and [26].

Let $G_{\circ}$ denote the class of closed convex subsets containing a point $x_{\circ}$ of a convex metric space $(X, d)$ with property (I). For $M \in G_{\circ}$ and $p \in X$, let $M_{p}=\left\{x \in M: d\left(x, x_{\circ}\right) \leq 2 d\left(p, x_{\circ}\right)\right\}$, let $P_{M}(p)=\{x \in M: d(p, x)=d(p, M)\}$ be the set of best approximants to $p$ in $M, C_{M}^{S}(p)=\{x \in$ $\left.M: S x \in P_{M}(p)\right\}$.

Proceeding as in Theorem 2.6 [4], we prove the following:

Theorem 2.7. Let $S$ and $T$ be self maps of a convex metric space $(X, d)$ with Property $(I), u \in$ $F(S) \cap F(T)$ and $M \in G_{\circ}$ such that $T\left(M_{u}\right) \subseteq S(M) \subseteq M$. Suppose that cl $\left(S\left(M_{u}\right)\right)$ is compact, $S$ is affine, $T$ is continuous on $M_{u}$ and satisfies $d(T x, u) \leq d(S x, u), d(S x, u) \leq d(x, u)$ for all $x \in M_{u}$. Then

(i) $P_{M}(u)$ is nonempty, closed and convex, 
(ii) $T\left(P_{M}(u)\right) \subseteq S\left(P_{M}(u)\right) \subseteq P_{M}(u)$, provided that $d(S x, S u)=d(x, u)$ for all $x \in C_{M}^{S}(u)$, and

(iii) $P_{M}(u) \cap F(S) \cap F(T) \neq \varnothing$ provided that $d(S x, S u)=d(x, u)$ for all $x \in C_{M}^{S}(u)$, S satisfies for some $q \in F(S)$,

$$
\begin{array}{r}
d(S x, S y) \leq \max \{d(x, y), c[\operatorname{dist}(x,[q, S y])+\operatorname{dist}(y,[q, S x])]\}+ \\
\frac{1-k}{k} \max \{\operatorname{dist}(x,[q, S x]), \operatorname{dist}(y,[q, y])\},
\end{array}
$$

for all $x, y \in P_{M}(u), 0 \leq c<\frac{1}{2}, k \in(0,1), c l T\left(P_{M}(u)\right) \subseteq S\left(P_{M}(u)\right), S$ and $T$ are $C_{q^{-}}$ commuting on $P_{M}(u)$, and $T$ satisfies for all $q \in F(S)$

$$
\begin{array}{r}
d(T x, T y) \leq \max \{d(S x, S y), c[\operatorname{dist}(S x,[q, T y])+\operatorname{dist}(S y,[q, T x])]\} \\
+\frac{1-k}{k} \max \{\operatorname{dist}(S x,[q, T x]), \operatorname{dist}(S y,[q, T y])\},
\end{array}
$$

for all $x, y \in P_{M}(u), 0 \leq c<\frac{1}{2}, k \in(0,1)$.

Proof. If $u \in M$ then all the arguments are obvious. So assume that $u \notin M$. If $x \in M \backslash M_{u}$, then $d\left(x, x_{\circ}\right)>2 d\left(u, x_{\circ}\right)$ and so $d(u, x) \geq d\left(x, x_{\circ}\right)-d\left(u, x_{\circ}\right)>d\left(u, x_{\circ}\right) \geq d i s t(u, M)$. Thus $\alpha=$ $\operatorname{dist}(u, M) \leq d\left(u, x_{\circ}\right)$. Since $\operatorname{cl}\left(S\left(M_{u}\right)\right)$ is compact, and the distance function is continuous, there exists $z \in \operatorname{cl}\left(S\left(M_{u}\right)\right)$ such that $\beta=\operatorname{dist}\left(u, \operatorname{cl}\left(S\left(M_{u}\right)\right)\right)=d(u, z)$. Hence

$$
\begin{aligned}
\alpha=\operatorname{dist}(u, M) & \leq \operatorname{dist}\left(u, \operatorname{cl}\left(S\left(M_{u}\right)\right)\right) \\
& =\beta \\
& \leq \operatorname{dist}\left(p, S\left(M_{u}\right)\right) \\
& \leq d(u, S x) \\
& \leq d(u, x)
\end{aligned}
$$

for all $x \in M_{u}$. Therefore $\alpha=\beta=\operatorname{dist}(u, M)$ i.e. $\operatorname{dist}(u, M)=\operatorname{dist}\left(u, c l\left(S\left(M_{u}\right)\right)\right)=d(u, z)$ i.e. $z \in P_{M}(u)$ and so $P_{M}(u)$ is nonempty. The closedness and convexity of $P_{M}(u)$ follows from that of $M$. This proves (i).

To prove (ii) let $z \in P_{M}(u)$. Then $d(S z, u)=d(S z, S u) \leq d(z, u)=\operatorname{dist}(u, M)$. This implies that $S z \in P_{M}(u)$ and so $S\left(P_{M}(u)\right) \subseteq P_{M}(u)$. Let $y \in T\left(P_{M}(u)\right)$. Since $T\left(M_{u}\right) \subseteq S(M)$ and $P_{M}(u) \subseteq$ $M_{u}$, there exists $z \in P_{M}(u)$ and $x_{1} \in M$ such that $y=T z=S x_{1}$. Further, we have $d\left(S x_{1}, u\right)=$ $d(T z, u) \leq d(S z, u) \leq d(z, u)=\operatorname{dist}(u, M)$. Thus $S x_{1} \in P_{M}(u)$ and $x_{1} \in C_{M}^{S}(u)$. Also, as $S x_{1} \in M$ and $\operatorname{dist}(u, M) \leq d\left(S x_{1}, u\right)$, it follws that $\operatorname{dist}(u, M)=d\left(S x_{1}, u\right)$. Since $d\left(x_{1}, u\right)=d\left(S x_{1}, u\right)=$ $\operatorname{dist}(u, M), x_{1} \in P_{M}(u)$ and $y=S x_{1} \in S\left(P_{M}(u)\right)$. Hence $T\left(P_{M}(u)\right) \subseteq S\left(P_{M}(u)\right)$ and so (ii) holds. The compactness of $\operatorname{cl}\left(S\left(M_{u}\right)\right)$ implies that $c l\left(S\left(P_{M}(u)\right)\right)$ is compact and hence complete. This, together with inequality (2.3) imply, by Theorem 2.4, that $P_{M}(u) \cap F(S) \neq \varnothing$. It follows that there exists a $q \in P_{M}(u)$ such that $q \in F(S)$. By (ii), the compactness of $\operatorname{cl}\left(S\left(M_{u}\right)\right)$ implies that $\operatorname{clT}\left(P_{M}(u)\right)$ is compact. Hence the conclusion (iii) follows from Theorem 2.4 applied to $P_{M}(u)$. 
Theorem 2.8. Let $S$ and $T$ be self maps of a convex metric space $(X, d)$ with Property $(I), u \in$ $F(S) \cap F(T)$ and $M \in G_{\circ}$ such that $T\left(M_{u}\right) \subseteq S(M) \subseteq M$. Suppose that $c l\left(T\left(M_{u}\right)\right)$ is compact, $S$ is affine, $T$ is continuous on $M_{u}, T, S$ satisfies $d(T x, u) \leq d(S x, u), d(S x, u) \leq d(x, u)$ for all $x \in M_{u}$. Then

(i) $P_{M}(u)$ is nonempty, closed and convex,

(ii) $T\left(P_{M}(u)\right) \subseteq S\left(P_{M}(u)\right) \subseteq P_{M}(u)$, provided that $d(S x, S u)=d(x, u)$ for all $x \in C_{M}^{S}(u)$, and

(iii) $P_{M}(u) \cap F(S) \cap F(T) \neq \varnothing$ provided that $d(S x, S u)=d(x, u)$ for all $x \in C_{M}^{S}(u), \operatorname{cl} T\left(P_{M}(u) \subseteq\right.$ $S\left(P_{M}(u)\right), S$ and $T$ are $C_{q}$-commuting on $P_{M}(u)$, and $T$ satisfies for some $q \in F(S)$

$$
\begin{array}{r}
d(T x, T y) \leq \max \{d(S x, S y), c[\operatorname{dist}(S x,[q, T y])+\operatorname{dist}(S y,[q, T x])]\} \\
+\frac{1-k}{k} \max \{\operatorname{dist}(S x,[q, T x]), \operatorname{dist}(S y,[q, T y])\},
\end{array}
$$

for all $x, y \in P_{M}(u), 0 \leq c<\frac{1}{2}, k \in(0,1)$.

Proof. The proof is similar to that of Theorem 2.7 .

Remark 2.3. Theorems 2.7 and 2.8 extend and generalize the corresponding results of [1], [2], [4], [12], [13], [18], [19], [20] and [22].

The following general common fixed point result will be needed in our next results.

Lemma 2.9 ([11]). Let $X$ be a Hausdorff topological space and $T$ and $I$ be continuous and nontrivially weakly compatible self maps of $X$. Then there exists a point $z$ in $X$ such that $T z=$ $I z=z$, provided $T$ satisfies the following condition:

$$
A \cap F(T) \neq \varnothing \text { for any } T \text {-invariant closed set } A \subset X \text {. }
$$

It is known (see[15]) that if $X$ is a Hausdorff topological space, $T$ a continuous self map of $X$ and if $T$ has relatively compact proper orbits, then $T$ satisfies condition (C).

Lemma 2.10. Let $M$ be a nonempty closed convex subset of a complete convex metric space $(X, d)$ with Property (I) and $T, f: M \rightarrow M$ are continuous and compatible. Suppose $T$ satisfies condition $(C), f$ is affine, $f(q)=q$ and $c l T(M) \subseteq f(M)$. If $c l T(M)$ is compact and the pair $(T, f)$ satisfies

$$
\begin{array}{r}
d(T x, T y) \leq \max \{d(f x, f y), c[\operatorname{dist}(f x,[q, T y])+\operatorname{dist}(f y,[q, T x])]\} \\
+\frac{1-k}{k} \max \{\operatorname{dist}(f x,[q, T x]), \operatorname{dist}(f y,[q, T y])\},
\end{array}
$$

for all $x, y \in M, 0 \leq c<\frac{1}{2}, k \in(0,1)$, then $T$ and $f$ have a common fixed point. 
Proof. Define $T_{n}$ as in Theorem 2.4. Proceeding as in Theorem 2.4, Corollary 2.2 guarantees that there exists an $x_{n} \in M$ such that $f x_{n}=T_{n} x_{n}$.

The compactness of $\operatorname{clT}(M)$ implies that there exists a subsequence $\left\{T x_{n_{i}}\right\}$ of $\left\{T x_{n}\right\}$ such that $T x_{n_{i}} \rightarrow y$. As $x_{n_{i}}=T_{n_{i}} x_{n_{i}}=W\left(T x_{n_{i}}, q, k_{n_{i}}\right) \rightarrow y$. The continuity of $f$ and $T$ imply that $T f x_{n_{i}} \rightarrow T y$ and $f T x_{n_{i}} \rightarrow f y$. By the compatibility of $f$ and $T$, we obtain $T y=f y$. Hence the pair $(T, f)$ is nontrivially compatible. Therefore, Lemma 2.9 implies that $M \cap F(f) \cap F(T) \neq$ $\varnothing$.

Proceeding as in Theorem 2.10 [4], we prove the following:

Theorem 2.11. Let $S$ and $T$ be self maps of a convex metric space $(X, d)$ with Property (I), $u \in F(S) \cap F(T)$ and $M \in G_{\circ}$ such that $T\left(M_{u}\right) \subseteq S(M) \subseteq M$. Suppose that $S$ is affine, continuous, $T$ is continuous on $M_{u}, T, S$ satisfies $d(T x, u) \leq d(S x, u), d(S x, u) \leq d(x, u)$ for all $x \in M_{u}$, and one of the following two conditions is satisfied:

(a) $\operatorname{cl}\left(S\left(M_{u}\right)\right)$ is compact,

(b) $\operatorname{cl}\left(T\left(M_{u}\right)\right)$ is compact.

Then

(i) $P_{M}(u)$ is nonempty, closed and convex,

(ii) $T\left(P_{M}(u)\right) \subseteq S\left(P_{M}(u)\right) \subseteq P_{M}(u)$, provided that $d(S x, S u)=d(x, u)$ for all $x \in C_{M}^{S}(u)$, and

(iii) $P_{M}(u) \cap F(S) \cap F(T) \neq \varnothing$ provided that $d(S x, S u)=d(x, u)$ for all $x \in C_{M}^{S}(u), S\left(P_{M}(u)\right)$ is closed, $S$ and $T$ satisfy condition $(C)$ on $P_{M}(u), S$ and $T$ are compatible on $P_{M}(u)$ and $T$ satisfies for some $q \in F(S)$

$$
\begin{array}{r}
d(T x, T y) \leq \max \{d(S x, S y), c[\operatorname{dist}(S x,[q, T y])+\operatorname{dist}(S y,[q, T x])]\} \\
+\frac{1-k}{k} \max \{\operatorname{dist}(S x,[q, T x]), \operatorname{dist}(S y,[q, T y])\},
\end{array}
$$

for all $x, y \in P_{M}(u), 0 \leq c<\frac{1}{2}, k \in(0,1)$.

Proof. The proof (i)-(ii) is similar to that of Theorem 2.7.

(iii) (a) By (i), $P_{M}(u)$ is closed, and by (ii), $P_{M}(u)$ is $S$-invariant, so by condition (C), $P_{M}(u) \cap F(S) \neq \varnothing$. It follows that there exists a $q \in P_{M}(u)$ such that $q \in F(S)$. By (ii), the compactness of $\operatorname{clS}\left(M_{u}\right)$ implies that of $\operatorname{clT}\left(P_{M}(u)\right)$. The conclusion now follows from Lemma 2.10 applied to $P_{M}(u)$.

(iii) b) By (i), $P_{M}(u)$ is closed, and by (ii), $P_{M}(u)$ is $S$-invariant, so by condition (C), $P_{M}(u) \cap$ $F(S) \neq \varnothing$. It follows that there exists a $q \in P_{M}(u)$ such that $q \in F(S)$. As the compactness of $\operatorname{clT}\left(M_{u}\right)$ implies that $\operatorname{clT}\left(P_{M}(u)\right)$ is compact. So, the conclusion follows from Lemma 2.10 applied to $P_{M}(u)$. 
Remark 2.4. (a) Theorem 2.11 extends the corresponding results of [1], [2], [4], [12], [13], [18], [19] and [22] to a compatible pair that is not necessarily nonexpansive and linear.

(b) Let $X=\mathbb{R}$ be endowed with usual metric and $M=[1, \infty)$. Let $S x=2 x-1$ and $T x=x^{2}$, for all $x \in M$. Let $q=1$. Then $M$ is convex, $q$-starshaped with $S q=q$ and $C_{q}(S, T)=[1, \infty)$. Here $S$ and $T$ are weakly compatible maps, $T$ satisfies condition (C), but they are not $C_{q}$-commuting.

\section{Acknowledgements}

The author is thankful to the learned referee for valuable suggestions.

\section{References}

[1] M. A. Al-Thagafi, Common fixed points and best approximation, J. Approx. Theory 85(1996), 318-323.

[2] M. A. Al-Thagafi and N. Shahzad, Noncommuting self maps and invariant approximations, Nonlinear Analysis 64 (2006), No. 12, 2778-2786.

[3] G. V. R. Babu and K. N. V. V. Vara Prasad, Common fixed point theorems of different compatible type mappings using Cirić's contraction type condition, Math. Comm. 11 (2006), 87-102.

[4] S. Chandok and T. D. Narang, Common fixed points and invariant approximation for Gregus type contraction mappings, Rendiconti Circolo Mat. Palermo, 60(2011), 203-214.

[5] L. Cirić, On a common fixed point theorem of a Gregus type, Publ. Inst. Math. 49(1991), 174-178.

[6] L. Cirić, On Diviccaro, Fisher and Sessa open questions, Arch. Math. (BRNO) 29(1993), 145-152.

[7] M. L. Diviccaro, B. Fisher, S. Sessa, A common fixed point theorem of Gregus type, Publ. Math. Debrecen 34(1987), 83-89.

[8] B. Fisher and S. Sessa, On a fixed point theorem of Gregus, Internat. J. Math. Math. 9(1986), 23-28.

[9] M. D. Guay, K. L. Singh and J. H. M. Whitfield, Fixed point theorems for nonexpansive mappings in convex metric spaces, Proc. Conference on nonlinear analysis (Ed. S.P.Singh and J. H. Bury) Marcel Dekker 80(1982), 179-189.

[10] M. Gregus, A fixed point theorem in Banach space, Boll. Un. Mat. Ital. (5) 7-A (1980), 193-198.

[11] M. Grinc and L. Snoha, Jungck theorem for triangular maps and related results, Appl. General Topology 1(2000), 83-92.

[12] L. Habiniak, Fixed point theorems and invariant approximation, J. Approx. Theory 56(1989), 241-244.

[13] N. Hussain, B. E. Rhoades and G. Jungck, Common fixed point snf invariant approximation results for Gregus type I-contractions, Num. Func. Anal. Optim. 28(2007), 1139-1151.

[14] G. Jungck, On a fixed point theorem of Fisher and Sessa, Internat. J. Math. Math. Sci. 13(1990), 497-500.

[15] G. Jungck, Common fixed point theorems for compatible self maps of Hausdorff topological spaces, Fixed Point Theory Appl. 3(2005), 355-363.

[16] G. Jungck and S. Sessa, Fixed point theorems in best approximation theory, Math. Japon. 42(1995), 249-252.

[17] R. N. Mukherjee and V. Verma, A note on a fixed point theorem of Gregus, Math. Japon. 33(1988), 745-749.

[18] T. D. Narang and S. Chandok, Fixed points of quasi-nonexpansive mappings and best approximation, Selçuk J. Appl. Math. 10(2009), 75-80.

[19] T. D. Narang and S. Chandok, Fixed points and best approximation in metric spaces, Indian J. Math. 51(2009), 293-303.

[20] T.D. Narang and S. Chandok, Common fixed points and invariant approximation of R-subweakly commuting maps in convex metric spaces, Ukrainian Math. J. 62(2010), 1367-1376.

[21] S. A. Sahab, M. S. Khan and S. Sessa, A result in best approximation theory, J. Approx. Theory 55(1988), 349351. 
[22] N. Shahzad, Invariant approximations and R-subweakly commuting maps, J. Math. Anal. Appl. 257(2001), 39-45.

[23] N. Shahzad, Noncommuting maps and best approximations, Rad. Math. 10(2001), 77-83.

[24] S. P. Singh, An application of fixed point theorem to approximation theory, J. Approx. Theory 25(1979), 89-90.

[25] A. Smoluk, Invariant approximations, Mat. Stos. 17(1981), 17-22.

[26] P.V. Subrahmanyam, An application of a fixed point theorem to best approximation, J. Approx. Theory 20(1977), 165-172.

[27] W. Takahashi, A convexity in metric space and nonexpansive mappings I, Kodai Math. Sem. Rep. 22(1970), 142-149.

Department of Mathematics, Khalsa College of Engineering \& Technology, Punjab Technical University, Ranjit Avenue, Amritsar-143001.

E-mail: chansok.s@gmail.com 